\title{
What if the masses of the first two quark families are not generated by the standard model Higgs boson?
}

\author{
F. J. Botella, ${ }^{1,3, *}$ G. C. Branco, ${ }^{2,3, \dagger}$ M. N. Rebelo, ${ }^{2,3, *}$ and J. I. Silva-Marcos ${ }^{2, \S}$ \\ ${ }^{1}$ Departament de Física Tè̀rica and IFIC, Universitat de València-CSIC, E-46100 Burjassot, Spain \\ ${ }^{2}$ Departamento de Física and Centro de Física Teórica de Partículas (CFTP), Instituto Superior Técnico \\ (IST), Universidade de Lisboa (UL), Avenida Rovisco Pais, P-1049-001 Lisboa, Portugal \\ ${ }^{3}$ Theory Department, CERN, CH 1211 Geneva 23, Switzerland
}

(Received 11 March 2016; published 29 December 2016)

\begin{abstract}
We point out that in the standard model there is meaningful quark mixing even in the extreme chiral (EC) limit, where only the third generation of quarks acquires mass. This mixing is in general expected to be of order 1 and the fact that $\left|V_{13}\right|^{2}+\left|V_{23}\right|^{2} \approx 1.6 \times 10^{-3}$ implies a novel fine-tuning problem in the SM which we point out for the first time. We propose a possible way of avoiding this fine-tuning by introducing a symmetry $S$ which leads to $V_{\mathrm{CKM}}=\mathbb{1}$, with only the third generation of quarks acquiring mass. We consider two scenarios for generating the mass of the first two quark generations and full quark mixing based on the assumption that the masses of the first two quark families are not generated by the standard Higgs. One consists of the introduction of a second Higgs doublet which is neutral under $S$. The second scenario consists of assuming new physics at a high energy scale, contributing to the masses of light quark generations, in an effective field theory approach. This last scenario leads to couplings of the Higgs particle to $s \bar{s}$ and $c \bar{c}$ which are significantly enhanced with respect to those of the SM. In both schemes, one has scalar-mediated flavor-changing neutral currents which are naturally suppressed. Flavor-violating top decays are predicted in the second scenario at the level $\operatorname{Br}(t \rightarrow h c) \geq 5 \times 10^{-5}$.
\end{abstract}

DOI: $10.1103 /$ PhysRevD.94.115031

\section{INTRODUCTION}

The recent discovery of the Higgs particle at the LHC rendered it even more urgent to understand the mechanism responsible for the generation of fermion masses and mixing. In the framework of the standard model (SM), fermion masses arise exclusively through Yukawa interactions and the Brout-Englert-Higgs mechanism. Some of the outstanding questions that one may ask include:

(i) Two of the salient flavor features in the quark sector are the strong hierarchy of quark masses and the fact that the $V_{\mathrm{CKM}}$ matrix is close to the identity. In the framework of the SM, can one conclude that these two features are related in some way? How can one understand small quark mixing in the SM?

(ii) In the SM, all fermion masses are generated through the vacuum expectation value (VEV) of the standard Higgs. Alternatively, one may consider a scenario where the standard Higgs only gives mass to the third generation, while the masses of the two first

\footnotetext{
*fbotella@uv.es

†branco@tecnico.ulisboa.pt

rebelo@tecnico.ulisboa.pt

juca@cftp.tecnico.ulisboa.pt
}

Published by the American Physical Society under the terms of the Creative Commons Attribution 4.0 International license. Further distribution of this work must maintain attribution to the author(s) and the published article's title, journal citation, and DOI. generations originate from another source. A crucial question is: how can this alternative scenario be tested at the LHC and future accelerators?

In this paper, we address the above two questions. With respect to (i), we show that actually in the SM the "natural" value of $\left(\left|V_{13}\right|^{2}+\left|V_{23}\right|^{2}\right)$ is of order 1, much larger than the observed experimental value of $1.6 \times 10^{-3}$. In order to address this question, we study in detail quark mixing in the extreme chiral (EC) limit, where only the third generation of quarks acquires mass, while $m_{d}, m_{s}, m_{u}, m_{c}$ remain massless. In fact, the smallness of $\left(\left|V_{13}\right|^{2}+\left|V_{23}\right|^{2}\right)$ may be interpreted as a hint from experiment, indicating that one should find a symmetry or a principle accounting for this fact. This implies in all generality a fine-tuning problem in the SM. The fact that there is flavor mixing even in the limit where the first two quark generations do not acquire mass has been previously discussed in specific frameworks, namely in the framework of supersymmetric theories with a U(2) flavor symmetry [1-3].

With respect to question (ii) we consider the possibility that in leading order the SM Higgs only gives mass to the third generation. This is achieved in a natural way through the introduction of a discrete symmetry $S$ which leads to quark mass matrices of rank 1, aligned in flavor space. We then conjecture that the generation of the mass of the first two generations arises from a different source. If this new source is just another Higgs doublet and if one assumes that the new doublet is neutral with respect to the symmetry $S$, then one is led to a flavor structure analogous to what one 
encounters in a class of models [4,5] known as BrancoGrimus-Lavoura (BGL), which have been extensively analyzed in the literature [6-12]. If, on the other hand, the new contribution arises in the framework of an effective field theory where the new physics (NP) particles have been integrated out, then assuming that this NP contribution is of order $m_{s}$ and $m_{c}$ in the down and up sectors, one can estimate the couplings of the Standard Higgs to $t \bar{t}, b \bar{b}, c \bar{c}$, $s \bar{s}$. It turns out that the couplings to $t \bar{t}, b \bar{b}$ do not differ much from those in the SM, but the couplings to $c \bar{c}, s \bar{s}$ are significantly enhanced with respect to those in the SM.

\section{MIXING IN THE EC LIMIT}

We analyze quark mixing in the EC limit, where the quark mass matrices $M_{d}$ and $M_{u}$ are rank 1 matrices generated by two independent Yukawa coupling matrices $Y_{d}, Y_{u}$. Therefore, $M_{d}, M_{u}$ can be written

$$
\begin{aligned}
& M_{d}=U_{L}^{d \dagger} \operatorname{diag}\left(0,0, m_{b}\right) U_{R}^{d}, \\
& M_{u}=U_{L}^{u \dagger} \operatorname{diag}\left(0,0, m_{t}\right) U_{R}^{u} .
\end{aligned}
$$

One does not lose generality by considering the specific ordering of $m_{b}, m_{t}$ in Eq. (1), since a permutation changing these positions can always be included in the unitary matrices $U_{L, R}^{d, u}$. The quark mixing matrix appearing in the charged weak interactions is given by $V^{0}=U_{L}^{u \dagger} U_{L}^{d}$ and it is at this stage an arbitrary mixing matrix. Taking into account that in the EC limit the first two generations are massless, one can make an arbitrary redefinition of the light quark masses through a unitary transformation of the type

$$
W_{u, d}=\left[\begin{array}{cc}
X_{u, d} & 0 \\
0 & 1
\end{array}\right]
$$

where $X_{u, d}$ are $2 \times 2$ unitary matrices. Under this transformation $V^{0}$ transforms as $V^{0} \rightarrow V^{\prime}=W_{u}^{\dagger} V W_{d}$. One has the freedom to choose $X_{u, d}$ at will to diagonalize the $2 \times 2$ upper left sector of $V^{\prime}$, leading to $\left|V_{12}^{\prime}\right|=\left|V_{21}^{\prime}\right|=0$. Unitarity of $V^{\prime}$ leads then to the constraint $V_{13}^{\prime *} V_{23}^{\prime}=0$. One can then choose, without loss of generality, $V_{13}^{\prime}=0$ and $V_{\mathrm{CKM}}$ becomes then an orthogonal matrix, with mixing only between the second and third generation, characterized by an angle $\alpha$, with $\left|V_{23}^{\prime}\right|=\left|V_{32}^{\prime}\right|=|\sin \alpha|$. The important point that we wish to emphasize is that this mixing in the EC limit of the SM is arbitrary. The smallness of $\left|V_{13}\right|^{2}+\left|V_{23}\right|^{2}$ in the SM, in general, cannot be related to the smallness of the mass ratios $m_{i}^{2} / m_{3}^{2}$, where $i=1,2$. Therefore, in the framework of the SM the observed smallness of $\left|V_{23}\right|=O\left(10^{-2}\right)$ provides a hint for the presence of a flavor symmetry. The appearance of a nontrivial mixing, even in the EC limit case, corresponds to a misalignment of the two mass matrices $M_{d}, M_{u}$.

\section{AN INVARIANT MEASURE OF ALIGNMENT}

Experimentally one encounters in the quark sector $V_{\mathrm{CKM}} \approx \mathbb{1}$ which corresponds to an alignment of the quark mass matrices in flavor space. It is useful to have an invariant measure of the mixing defined in terms of the mass matrices when they are written in an arbitrary weak basis. This can be done by defining the following weak basis invariant [13]:

$$
A \equiv \frac{1}{2} \operatorname{tr} B^{2}, \quad \text { with } \quad B=h_{d}-h_{u}
$$

where the building blocks are the two matrices

$$
h_{d}=\frac{H_{d}}{\operatorname{tr}\left[H_{d}\right]}, \quad h_{u}=\frac{H_{u}}{\operatorname{tr}\left[H_{u}\right]}
$$

with the notation $H_{u, d} \equiv M_{u, d} M_{u, d}^{\dagger}$. By construction, one has $\operatorname{tr}\left[h_{d}\right]=\operatorname{tr}\left[h_{u}\right]=1$. Given the two rank 1 matrices $M_{d, u}$ described before, corresponding to the EC limit, one obtains

$$
A \equiv \frac{1}{2} \operatorname{tr} B^{2}=\left|V_{23}\right|^{2}+\left|V_{13}\right|^{2} .
$$

The result of Eq. (5) is exact in the EC limit. In this limit, this invariant $A$ varies from 0 to 1 , with 0 corresponding to exact alignment and 1 to total misalignment. The invariant $A$ still gives a measure of the size of mixing when the first two generations acquire mass, and in this case we have $A \approx\left|V_{23}\right|^{2}+\left|V_{13}\right|^{2}+O\left(m_{s} / m_{b}\right)^{4}$.

\section{OBTAINING SMALL MIXING THROUGH A SYMMETRY}

As stated before, mixing in the EC limit is parametrized by an arbitrary mixing angle involving two generations. In general, in the SM there is no reason to assume that this mixing angle is either close to zero or maximal; in fact it can take any value. It is possible to introduce a symmetry which leads to the vanishing of this mixing. Without loss of generality, this angle can parametrize mixing between the second and the third generations. Let us consider the following symmetry $S$, in the context of the particle content of the SM, with only one Higgs doublet:

$$
\begin{aligned}
Q_{L 3}^{0} & \rightarrow \exp (i \tau) Q_{L 3}^{0}, \quad u_{R 3}^{0} \rightarrow \exp (i 2 \tau) u_{R 3}^{0}, \\
\phi & \rightarrow \exp (i \tau) \phi, \quad \tau \neq 0, \pi
\end{aligned}
$$

where $Q_{L j}^{0}$ is a left-handed quark doublet and $\phi$ is the Higgs doublet. All other fermions transform trivially under $S$. This symmetry leads to the following pattern of texture zeros for the Yukawa couplings: 


$$
Y_{d}=\left[\begin{array}{ccc}
0 & 0 & 0 \\
0 & 0 & 0 \\
\times & \times & \times
\end{array}\right], \quad Y_{u}=\left[\begin{array}{lll}
0 & 0 & 0 \\
0 & 0 & 0 \\
0 & 0 & \times
\end{array}\right]
$$

which clearly lead to $V_{\mathrm{CKM}}$ equal to the identity. The matrices of Eq. (7) are written in the weak basis (WB) chosen by the symmetry. The matrix $Y_{d}$ can be written in the same form as $Y_{u}$ by means of a rotation of the righthanded down quarks, which simply corresponds to a different choice of WB.

Next we present possible ways of extending this scenario in order to generate the masses of the first two generations of quarks, as required experimentally, without generating large mixing and keeping the Higgsmediated flavor-changing neutral currents (HFCNC) under control.

\section{GENERATING THE MASSES OF THE FIRST TWO QUARK GENERATIONS}

At this stage, one has to address the question of the origin of the masses of the first two generations. The discovery of the Higgs particle at the LHC and the study of its production and decay has shown that the VEV of the Higgs field gives the dominant contribution to the masses of the fermions of the third generation, namely to the top and bottom quarks, as well as the $\tau$-lepton. It is conceivable that the masses of the quarks of the first two generations arise from a different source [14-20] so that the quark mass matrices have the form

$$
M=M^{(0)}+M^{(1)}
$$

where $M^{(0)}$ is generated by the VEV of the standard Higgs $\phi$ and $M^{(1)}$ may arise from the VEV of a second Higgs $\phi^{\prime}$ or from another unspecified source. In either case, the fact that there are two different sources giving contributions to the mass of quarks of a given charge leads to scalar-mediated flavor-changing neutral currents (FCNC). These currents are naturally suppressed in both of the scenarios we consider below, once the experimental values of the $V_{\mathrm{CKM}}$ entries are taken into account.

\section{ADDING A SECOND HIGGS DOUBLET}

The simplest possibility to generate masses for the first two generations is in the context of two-Higgs-doublet models [21]. If the second doublet $\phi^{\prime}$ is neutral under $S$, the implied structure of the Yukawa couplings of $\phi^{\prime}$ is

$$
Y_{d}^{\prime}=\left[\begin{array}{ccc}
\times & \times & \times \\
\times & \times & \times \\
0 & 0 & 0
\end{array}\right] ; \quad Y_{u}^{\prime}=\left[\begin{array}{ccc}
\times & \times & 0 \\
\times & \times & 0 \\
0 & 0 & 0
\end{array}\right] .
$$

Since we now have two Higgs doublets, the Yukawa couplings written in terms of quark mass eigenstates $u$, $d$ are

$$
\begin{aligned}
L_{Y}= & -\frac{\sqrt{2} H^{+}}{v} \bar{u}\left(V N_{d} \gamma_{R}-N_{u}^{\dagger} V \gamma_{L}\right) d+\text { H.c. } \\
& -\frac{H^{0}}{v}\left(\bar{u} D_{u} u+\bar{d} D_{d} d\right) \\
& -\frac{R}{v}\left[\bar{u}\left(N_{u} \gamma_{R}+N_{u}^{\dagger} \gamma_{L}\right) u+\bar{d}\left(N_{d} \gamma_{R}+N_{d}^{\dagger} \gamma_{L}\right) d\right] \\
& +i \frac{I}{v}\left[\bar{u}\left(N_{u} \gamma_{R}-N_{u}^{\dagger} \gamma_{L}\right) u-\bar{d}\left(N_{d} \gamma_{R}-N_{d}^{\dagger} \gamma_{L}\right) d\right]
\end{aligned}
$$

where $v \equiv \sqrt{v_{1}^{2}+v_{2}^{2}} \approx 246 \mathrm{GeV}, \gamma_{L}=\left(1-\gamma_{5}\right) / 2, \gamma_{R}=$ $\left(1+\gamma_{5}\right) / 2$ and $V$ denotes the $V_{\mathrm{CKM}}$ matrix. The neutral scalar fields $H^{0}, R$ are orthogonal combinations of the fields $\rho_{j}$, introduced when one expands the neutral scalar fields around their VEVs, $\phi_{j}^{0}=\frac{e^{i \alpha_{j}}}{\sqrt{2}}\left(v_{j}+\rho_{j}+i \eta_{j}\right)$, where we have opted for the notation $\phi_{1} \equiv \phi, \phi_{2} \equiv \phi^{\prime}$. Analogously $I$ stands for the linear combination of the $\eta_{j}$ orthogonal to the neutral Goldstone boson. The physical neutral Higgs fields correspond to linear combinations of $H^{0}, R$ and $I$. The Yukawa couplings of Eq. (9) lead to a contribution to the quark mass matrices of the form

$$
\begin{aligned}
& M_{d}^{(1)}=\frac{v^{\prime}}{\sqrt{2}}\left[\begin{array}{lll}
\times & \times & \times \\
\times & \times & \times \\
0 & 0 & 0
\end{array}\right] ; \\
& M_{u}^{(1)}=\frac{v^{\prime}}{\sqrt{2}}\left[\begin{array}{lll}
\times & \times & 0 \\
\times & \times & 0 \\
0 & 0 & 0
\end{array}\right] .
\end{aligned}
$$

In general two-Higgs doublet models (2HDM) the couplings parametrized by the flavor matrices $N_{d}, N_{u}$ in Eq. (10) lead to FCNC. However, it has been shown that in BGL models [4] the full flavor structure of $N_{d}, N_{u}$ only depends on the $V_{\mathrm{CKM}}$ matrix. This structure of Eq. (11) coincides with what one encounters in a class of BGL models [4]. In this model there are FCNC but they are naturally suppressed by small $V_{\mathrm{CKM}}$ elements and the model obeys the minimal flavor violation $[5,22,23]$ principle.

In this context, there are two types of BGL models: (1) top models described by Eqs. (6), (7) and (11) with FCNC only in the down sector and (2) bottom models with the role of up and down quarks interchanged. This second class of models gives rise to FCNCs only in the up sector. From low energy flavor data the scale of new physics in top models can be quite light at a few hundred $\mathrm{GeV}$ [9]. Bottomlike models introduce new scales close to the $\mathrm{TeV}$ region [9]. Flavor-conserving or flavor-blind Higgs observables can be accommodated in both categories because the 
new couplings, compared to the SM couplings, i.e., the coupling modifiers $\kappa_{Z}, \kappa_{W}, \kappa_{t}, \kappa_{\tau}, \kappa_{b}, \kappa_{g}$ and $\kappa_{\gamma}$ may deviate from 1 at the percent level. These models have been extensively studied in the literature [8-12]. In the Higgs sector the most relevant prediction specific to top models is the decay $h \rightarrow b \bar{s}+s \bar{b}$ with branching ratios at most between $10^{-3}$ and $10^{-2}$ [11]. The bottom models predict the rare top decay $t \rightarrow h c$ with a branching ratio of at most $10^{-3}$ [11]. In both classes of models these predictions can be correlated with $h \rightarrow \mu \bar{\tau}+\tau \bar{\mu}$ occurring at a branching ratio which can reach at most $10^{-2}$.

\section{GENERATING LIGHT QUARK MASSES FROM NEW PHYSICS AT A HIGH ENERGY SCALE}

Here, we consider that only one Higgs doublet is introduced in the framework of the SM and introduce the symmetry $S$ of Eq. (6) which implies that only the third generation of quarks acquire mass, with $V_{\mathrm{CKM}}=1$. We shall consider that the quark masses of the first two generations arise from new physics contributing to the Yukawa couplings in leading order through an effective dimension-six operator of the form

$$
\begin{aligned}
\mathcal{L}_{\text {eff }}= & -\left(Y_{d}^{(1)}\right)_{j k} \frac{\phi^{\dagger} \phi}{\Lambda^{2}} \bar{Q}_{L_{j}}^{0} d_{R_{k}}^{0} \phi \\
& -\left(Y_{u}^{(1)}\right)_{j k} \frac{\phi^{\dagger} \phi}{\Lambda^{2}} \bar{Q}_{L_{j}}^{0} u_{R_{k}}^{0} \tilde{\phi} .
\end{aligned}
$$

The Yukawa and quark mass matrices have then the form [16]

$$
\begin{aligned}
\sqrt{2} Y_{d, u} & =Y_{d, u}^{(0)}+3 Y_{d, u}^{(1)}\left(\frac{v^{2}}{\Lambda^{2}}\right) \\
M_{d, u} & =v\left[Y_{d, u}^{(0)}+Y_{d, u}^{(1)}\left(\frac{v^{2}}{\Lambda^{2}}\right)\right]
\end{aligned}
$$

with $Y_{d}^{(0)}=\operatorname{diag}\left(0,0, \frac{m_{b}}{v}\right)$ and $Y_{u}^{(0)}=\operatorname{diag}\left(0,0, \frac{m_{t}}{v}\right)$. The fact that $Y_{d, u}$ are not proportional to $M_{d, u}$ leads to Higgs-mediated FCNCs. At this stage, it is useful to estimate the size of the new mass scale $\Lambda$. From Eq. (13) and taking into account that $v=174 \mathrm{GeV}, m_{t}=$ $173 \mathrm{GeV}$ one obtains $\left(Y_{u}^{(0)}\right)_{t t} \approx 1$. Assuming $Y_{u}^{(1)} \approx\left(Y_{u}^{(0)}\right)_{t t}$, one obtains $\Lambda=\left[\frac{Y_{u}^{(1)} v^{3}}{m_{c}}\right]^{1 / 2} \approx 2 \mathrm{TeV}$, so the new mass scale is of the order of a few TeV. For the down quark sector, taking into account that $m_{b} \approx 4.2 \mathrm{GeV}, m_{s} \approx 0.095 \mathrm{GeV}$, one obtains $\left(Y_{d}^{(0)}\right)_{b b} \approx \frac{m_{b}}{v} \approx 0.02, Y_{d}^{(1)} \approx 0.07$. Note that in the present framework one obtains $\left|V_{23}\right| \approx O\left(m_{s} / m_{b}\right)$ but one does not provide an explanation for the smallness of $m_{b} / m_{t}$. We will show that the potentially dangerous FCNCs are naturally suppressed in the present framework. The down quark mass matrix is diagonalized by

$$
U_{d L}^{\dagger}\left[Y_{d}^{(0)}+Y_{d}^{(1)} \frac{v^{2}}{\Lambda^{2}}\right] U_{d R}=\frac{D_{d}}{v}
$$

where $D_{d} \equiv \operatorname{diag}\left(m_{d}, m_{s}, m_{b}\right)$, with an analogous expression for the up sector. In the quark mass eigenstate basis, the Yukawa coupling matrix becomes

$$
\sqrt{2} Y_{d}^{m}=\sqrt{2}\left(U_{d L}^{\dagger} Y_{d} U_{d R}\right)=\frac{3 D_{d}}{v}-2 U_{d L}^{\dagger} Y_{d}^{(0)} U_{d R} .
$$

At this stage, it is useful to write $U_{d L}^{\dagger} Y_{d}^{(0)} U_{d R}$ explicitly. Taking into account the form of $Y_{d}^{(0)}$ one obtains

$$
\left(U_{d L}^{\dagger} Y_{d}^{(0)} U_{d R}\right)_{j k}=\left(U_{d L}^{*}\right)_{3 j}\left(U_{d R}\right)_{3 k} \frac{m_{b}}{v}
$$

with an analogous expression for the up sector. The strength of the Higgs couplings $Y_{d}^{m}$ is controlled by Eqs. (15), (16) and one has to take into account the very strict bounds on flavor-violating scalar couplings, which can be derived from $K^{0}-\bar{K}^{0}, B_{d}-\bar{B}_{d}, B_{s}-\bar{B}_{s}, D^{0}-\bar{D}^{0}$ mixings. These bounds have been recently analyzed in [24]. From $B_{s}-\bar{B}_{s}$ mixing, one derives bounds on $\left|\left(U_{d L}^{*}\right)_{32}\left(U_{d R}\right)_{33}\right|$ and $\left|\left(U_{d L}^{*}\right)_{33}\left(U_{d R}\right)_{32}\right|$ which taking into account that $\left|\left(U_{d L}\right)_{33}\right| \approx 1$ and $\left|\left(U_{d R}\right)_{33}\right| \approx 1$ lead to $\left|\left(U_{d L}\right)_{32}\right| \leq 1.4 \times 10^{-2}$. Similarly, one derives from $B_{d}-\bar{B}_{d}$ mixing the bound $\left|\left(U_{d L}\right)_{31}\right| \leq 3 \times 10^{-3}$. It is remarkable that these bounds lead to $\left|\left(U_{d L}^{*}\right)_{31}\left(U_{d R}\right)_{32}\right| \simeq$ $\left|\left(U_{d L}^{*}\right)_{32}\left(U_{d R}\right)_{31}\right| \leq 4.4 \times 10^{-4}$ which guarantees that the Higgs contribution to $K^{0}-\overline{K^{0}}$ mixing is sufficiently suppressed, to conform to the strict experimental bound. Flavor-changing scalar couplings in the up sector are controlled by $\left|\left(U_{u L}^{*}\right)_{3 i}\left(U_{u R}\right)_{3 j}\right|$. On the other hand, $U_{u L}$ is constrained to be in a region which can generate the observed $V_{\mathrm{CKM}}=\left(U_{u L}^{\dagger} U_{d L}\right)$. Once these constraints are taken into account, one predicts, in the present framework, the strength of flavor-changing decays of the top quark, namely

$$
\operatorname{Br}(t \rightarrow h c) \geq 5 \times 10^{-5} .
$$

It is interesting to notice that in this framework the previously analyzed new flavor-changing Higgs contributions all arise from the third column of the matrices $U_{d L}$, $U_{d R}, U_{u L}$ and $U_{u R}$.

So far we have only discussed the off-diagonal Higgs couplings. In the diagonal couplings, one has to distinguish between the couplings of the third generation (i.e. $t \bar{t} h$ and $b \bar{b} h$ ) and those of the two light generations. Taking into account that $\left|\left(U_{d L}^{*}\right)_{33}\left(U_{d R}\right)_{33}\right| \approx 1$ and also $\left|\left(U_{u L}^{*}\right)_{33}\left(U_{u R}\right)_{33}\right| \approx 1$ it is clear that the couplings of the third quark generation coincide with those in the SM. On the contrary, for the first two generations, one has a significant enhancement by a factor of 3 , leading, for example, to 
WHAT IF THE MASSES OF THE FIRST TWO QUARK ...

$$
\Gamma(h \rightarrow q \bar{q}) \approx 9 \Gamma^{\mathrm{SM}}(h \rightarrow q \bar{q}) \quad q=d, s, c .
$$

At this stage, the following comment is in order. For the down sector, the experimental constraints from meson mixing are very strict and the prediction of Eq. (18) for $q=d, s$, is solid. For $c \bar{c}$ although the enhancement of Eq. (18) holds for most of the allowed parameter space, there are regions of allowed parameter space where the enhancement is not as strong. So far, we have only discussed the quark sector. Note that the observed lepton flavor mixing is large and therefore there is no motivation to opt for the symmetry $S$ to act in the lepton sector in a way analogous to the quark sector, since this would lead to $V_{\text {PMNS }}=\mathbb{1}$ in leading order, in contrast to experiment. We shall assume that leptons are neutral with respect to $S$, which leads to couplings of the Higgs particle to leptons which coincide with those of the SM.

Taking into account that $\Gamma^{\mathrm{SM}}(h \rightarrow \bar{c} c) / \Gamma^{\mathrm{SM}}(h \rightarrow$ all $) \sim 3 \%$, and assuming Eq. (18) and that the other relevant decay channels do not change, we get $\Gamma(h \rightarrow$ all $) \approx$ $1.23 \Gamma^{\mathrm{SM}}(h \rightarrow$ all $)$. This result gives rise to a definitive prediction for the signal strength parameters $\mu^{f}$ [25] in the decay channels $f=\gamma \gamma, Z Z, W W, \tau \bar{\tau}, b \bar{b}$ of

$$
\mu^{f}=\frac{\Gamma(h \rightarrow f) \Gamma^{\mathrm{SM}}(h \rightarrow \text { all })}{\Gamma(h \rightarrow \text { all }) \Gamma^{\mathrm{SM}}(h \rightarrow f)} \approx 0.81,
$$

which is compatible with the combined ATLAS CMS analysis [25]. Looking at the coupling modifiers analysis $\kappa_{f}$ we have as in the SM no modification of the couplings to the relevant channels

$$
\kappa_{Z}=\kappa_{W}=\kappa_{t}=\kappa_{\tau}=\kappa_{b}=\kappa_{g}=\kappa_{\gamma}=1,
$$

but the large enhancement in the undetected $c \bar{c}$ channel contributes to the so-called beyond the SM branching ratio $\mathrm{BR}_{\mathrm{BSM}} \sim 18.8 \%$ in perfect agreement with the $34 \%$ joint upper bound from ATLAS and CMS at 95\% C.L. [25].

\section{TeV COMPLETION}

A possible $\mathrm{TeV}$ completion of the present model can be implemented in the framework of an extension of the SM where one adds three $Q=-1 / 3$ vectorlike quarks, $D_{\alpha}$, and three $Q=2 / 3$ vectorlike quarks, $U_{\beta}$, isosinglets of $S U(2)$, to the spectrum of the SM.

We introduce the symmetry $S$ considered in Eq. (6), with the standardlike quarks transforming as before. The symmetry $S$ is spontaneously broken by the VEV of the Higgs field and we also allow for a soft-breaking term $\bar{D}_{L \beta} d_{R 3}$ with a similar soft-breaking term for the up sector. In Fig. 1, we show a diagram contributing, in the down sector, to the leading higher order operators in Eq. (12).

The challenge of getting a detailed description of how to generate a realistic quark mass spectrum and a
PHYSICAL REVIEW D 94, 115031 (2016)

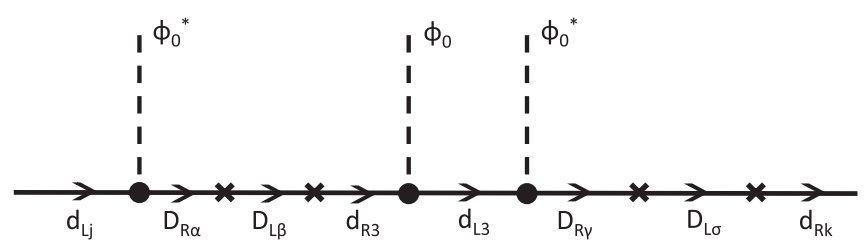

FIG. 1. Example of generation of effective mass terms for the light down quarks after spontaneous symmetry breaking, with only one soft $S$ breaking term involving the $R$ component of the third generation down quark.

correct pattern of quark mixing goes beyond the scope of this paper.

\section{CONCLUSIONS}

The crucial points of our paper are the following:

(i) Contrary to what may be a common belief, in the $\mathrm{SM}$, the natural value of $\left|V_{23}\right|^{2}+\left|V_{13}\right|^{2}$ is of order 1 . In the SM, without an additional symmetry, the smallness of $V_{\mathrm{CKM}}$ mixings cannot be derived from the observed strong hierarchy of quark masses.

(ii) We point out that the fact that $\left|V_{23}\right|^{2}+\left|V_{13}\right|^{2}$ is small may be considered as a hint of nature, suggesting the introduction of a symmetry $S$. We have given an example of such a symmetry, which leads to $V_{\mathrm{CKM}}=\mathbb{1}$, with only the third quark generation acquiring mass.

(iii) We have suggested two different scenarios to generate the masses of the two lighter quark generations. One of them consists of the introduction of a second Higgs doublet, which is neutral under $S$. This framework leads to a BGL-type model [4,5] which hase been analyzed in the literature. Another scenario consists of assuming that new physics at a high energy scale contributes to the light quark masses in an effective field theory approach. This scenario leads to the following striking predictions which can be tested at LHC run 2, as well as in other future accelerators: The diagonal Higgs quark couplings of the third generation, i.e. $t$ th and $b b h$, essentially coincide with those of the SM. The diagonal Higgs couplings of the lighter quarks are enhanced with respect to those of the SM, by about a factor of 3 , with the most significant effect of this enhancement given by Eq. (18). In this framework one predicts Higgs-mediated flavor-violating top decays, as indicated in Eq. (17).

(iv) In this paper we point out a fine-tuning problem that arises in the context of the SM and propose scenarios to solve the problem which lead to predictions that can be observed in the near future. If such scenarios are not realized in nature this fine-tuning problem remains an open question. Attempts at solving this question may lead to novel approaches to address the flavor puzzle. 


\section{ACKNOWLEDGMENTS}

The authors thank the CERN Theory Department for hospitality and partial financial support. We thank Luca Fiorini for interesting discussions. This work is partially supported by Spanish Ministerio de Economía y Competitividad (MINECO) under Grants No. FPA201568318-R and No. SEV-2014-0398, by Generalitat Valenciana under Grant No. GVPROMETEOII 2014-049 and by Fundação para a Ciência e a Tecnologia (FCT,
Portugal) through the Projects No. CERN/FIS-NUC/0010/ 2015 and CFTP-FCT Unit 777 (UID/FIS/00777/2013) which are partially funded through Programa Operacional Ciência Tecnologia Inovação (Fundo Europeu de Desenvolvimento Regional, FEDER), Programa Operacional Competitividade e Internacionalização, Quadro de Referência Estratégica Nacional and European Union. The authors also acknowledge the hospitality of Universidad de Valencia, IFIC, and CFTP at IST Lisboa during visits for scientific collaboration.
[1] A. Pomarol and D. Tommasini, Horizontal symmetries for the supersymmetric flavor problem, Nucl. Phys. B466, 3 (1996).

[2] O. Gedalia and G. Perez, Flavor physics, arXiv:1005.3106.

[3] R. Barbieri, G. Isidori, J. Jones-Perez, P. Lodone, and D. M. Straub, $U(2)$ and minimal flavour violation in supersymmetry, Eur. Phys. J. C 71, 1725 (2011).

[4] G. C. Branco, W. Grimus, and L. Lavoura, Relating the scalar flavor changing neutral couplings to the CKM matrix, Phys. Lett. B 380, 119 (1996).

[5] F. J. Botella, G. C. Branco, and M. N. Rebelo, Minimal flavour violation and multi-Higgs models, Phys. Lett. B 687, 194 (2010).

[6] F. J. Botella, G. C. Branco, M. Nebot, and M. N. Rebelo, Two-Higgs leptonic minimal flavour violation, J. High Energy Phys. 10 (2011) 037.

[7] F. J. Botella, G. C. Branco, and M. N. Rebelo, Invariants and flavour in the general two-Higgs doublet model, Phys. Lett. B 722, 76 (2013).

[8] G. Bhattacharyya, D. Das, P. B. Pal, and M. N. Rebelo, Scalar sector properties of two-Higgs-doublet models with a global U(1) symmetry, J. High Energy Phys. 10 (2013) 081.

[9] F. J. Botella, G. C. Branco, A. Carmona, M. Nebot, L. Pedro, and M. N. Rebelo, Physical constraints on a class of two-Higgs doublet models with FCNC at tree level, J. High Energy Phys. 07 (2014) 078.

[10] G. Bhattacharyya, D. Das, and A. Kundu, Feasibility of light scalars in a class of two-Higgs-doublet models and their decay signatures, Phys. Rev. D 89, 095029 (2014).

[11] F. J. Botella, G. C. Branco, M. Nebot, and M. N. Rebelo, Flavour changing Higgs couplings in a class of two Higgs doublet models, Eur. Phys. J. C 76, 161 (2016).

[12] M. Sher and K. Thrasher, Flavor changing leptonic decays of heavy Higgs bosons, Phys. Rev. D 93, 055021 (2016).
[13] G. C. Branco and J. I. Silva-Marcos, Invariants, alignment and the pattern of fermion masses and mixing, Phys. Lett. B 715, 315 (2012).

[14] K. S. Babu and S. Nandi, Natural fermion mass hierarchy and new signals for the Higgs boson, Phys. Rev. D 62, 033002 (2000).

[15] G. F. Giudice and O. Lebedev, Higgs-dependent Yukawa couplings, Phys. Lett. B 665, 79 (2008).

[16] A. Goudelis, O. Lebedev, and J. h. Park, Higgs-induced lepton flavor violation, Phys. Lett. B 707, 369 (2012).

[17] G. Perez, Y. Soreq, E. Stamou, and K. Tobioka, Constraining the charm Yukawa and Higgs-quark coupling universality, Phys. Rev. D 92, 033016 (2015).

[18] W. Altmannshofer, S. Gori, A. L. Kagan, L. Silvestrini, and J. Zupan, Uncovering mass generation through Higgs flavor violation, Phys. Rev. D 93, 031301 (2016).

[19] D. Ghosh, R. S. Gupta, and G. Perez, Is the Higgs mechanism of fermion mass generation a fact? A Yukawa-less firsttwo-generation model, Phys. Lett. B 755, 504 (2016).

[20] M. Bauer, M. Carena, and K. Gemmler, Creating the fermion mass hierarchies with multiple Higgs bosons, arXiv:1512.03458.

[21] For a review see G. C. Branco, P. M. Ferreira, L. Lavoura, M. N. Rebelo, M. Sher, and J. P. Silva, Theory and phenomenology of two-Higgs-doublet models, Phys. Rep. 516, 1 (2012).

[22] A. J. Buras, P. Gambino, M. Gorbahn, S. Jager, and L. Silvestrini, Universal unitarity triangle and physics beyond the standard model, Phys. Lett. B 500, 161 (2001).

[23] G. D’Ambrosio, G. F. Giudice, G. Isidori, and A. Strumia, Minimal flavor violation: An effective field theory approach, Nucl. Phys. B645, 155 (2002).

[24] G. Blankenburg, J. Ellis, and G. Isidori, Flavour-changing decays of a $125 \mathrm{GeV}$ Higgs-like particle, Phys. Lett. B 712, 386 (2012).

[25] Report Nos. ATLAS-CONF-2015-044 and CMS-PAS-HIG15-002. 